\title{
Thermodynamics of the QCD transition from lattice
}

\author{
Szabolcs Borsányi (for the Wuppertal-Budapest Collaboration) ${ }^{1}$ \\ Theoretische Physik, Bergische Universität Wupperal, D-42119, Germany
}

\begin{abstract}
We discuss recent developments in lattice QCD for the bulk thermodynamics of the transition. We review the current status of the equation of state, the possible implications of a magnetic field and the fluctuations of conserved charges, like the net electric charge or baryon number. We also show predictions for higher cumulants, which will be experimentally available in near future.
\end{abstract}

\section{Introduction}

Lattice simulations aiming to describe the features of quark gluon plasma and its transition to confined matter have become a field of high precision in the past years. An important goal of the efforts on the lattice is to provide theoretical background for collision experiments. The relevance of lattice results are most prominent for the heavy ion physics pursued at RHIC and LHC, where matter with high energy density, but moderate or small chemical potentials are produced.

Lattice simulations solve Quantum Chromodynamics (QCD) in equilibrium. The solution emerges from a continuum limit, which is an extrapolation from a set of simulations on lattices with smaller and smaller lattice spacings $(a)$. Finite temperature $(T)$ is naturally implemented by a torus in Euclidean time. In most thermodynamics studies the lattice spacing is expressed through the number of lattice points in the Euclidean time direction: $N_{t}$, which translates to $a=1 /\left(N_{t} T\right)$. In most early papers $N_{t}$ was set to 4 or 6 . By today $N_{t}=16$ data sets are not uncommon, and are believed to be necessary for a controlled continuum extrapolation even with improved actions.

Longer correlations (inverse pion mass) require larger computer resources to simulate the theory. In the past decade lattice field theory has seen a great development in gradually reducing the quark masses down to their physical values [1]. Simultaneously to this trend our increasing understanding of various discretization effects has lead to more efficient discretization schemes (actions) that allow a continuum extrapolation at an affordable cost [2, 3]. In view of these developments here we only discuss results with physical (or nearly physical) quark masses close to or in the continuum limit.

\section{Transition temperature}

Evidence form lattice QCD shows that at zero chemical potential the transition from the chirally broken, confining phase of hadrons to the quark gluon plasma phase is a crossover [4].

\footnotetext{
${ }^{1}$ borsanyi@uni-wuppertal.de

Preprint submitted to Nuclear Physics A
} 
The transition temperature of a crossover transition depends on the observable one chooses. At vanishing light quark masses the chiral condensate is a genuine order parameter, which can be used to extract a chiral transition temperature. At sufficiently high quark masses the deconfinement transition is identified by a discontinuity in the Polyakov loop as a function of temperature. Varying the quark mass in the space of theories the relevance of chiral and deconfinement aspects can be tuned. With sufficiently light $u$ and $d$ quarks an $\mathrm{O}(4)$ scaling of the chiral observables is conjectured [5, 6]. This universal mass dependence has been used to relate the transition temperature $\left(T_{c}\right)$ in QCD to $T_{c}$ for the actual simulation parameters [7].

The Wuppertal-Budapest collaboration has simulated the QCD transition with physical quark masses in the stout staggered formulation [8]. The lattice resolutions range from $N_{t}=6$ to $N_{t}=$ 16. From the inflection point of the chiral condensate we find $T_{c}=155(3)(3) \mathrm{MeV}$, where the first of the errors is statistical, the second is systematic, including the uncertainties of the scale setting [9, 10]. Other $T_{c}$ definitions from chiral observables span a range of $147-157 \mathrm{MeV}$. Recently the HotQCD collaboration has published a transition temperature, 154(8)(1) MeV, based on the $\mathrm{O}(4)$ scaling [7], which is very well compatible with the range given by the Wuppertal-Budapest collaboration in 2006. This is in contrast to the earlier continuum result $(192(4)(7) \mathrm{MeV}$ ) by the RBC-Bielefeld group. A key improvement since then was the adoption of the HISQ action [3].

The quoted values of $T_{c}$ are all based on lattice observables that are remnants of the chiral order parameter. One can also study other observables, such as the Polyakov loop, which is the remnant order parameter of the deconfinement transition. The Polyakov loop as well as the inflection point is renormalization scheme dependent. E.g. the scheme used in Ref. [9] was different than in our latest work [10]. We made this change in our convention to be compatible with Ref. [11] in every detail. While the comparison of our result to the asqtad data has showed a disagreement, the new HISQ data of the HotQCD collaboration was in agreement with ours [7].
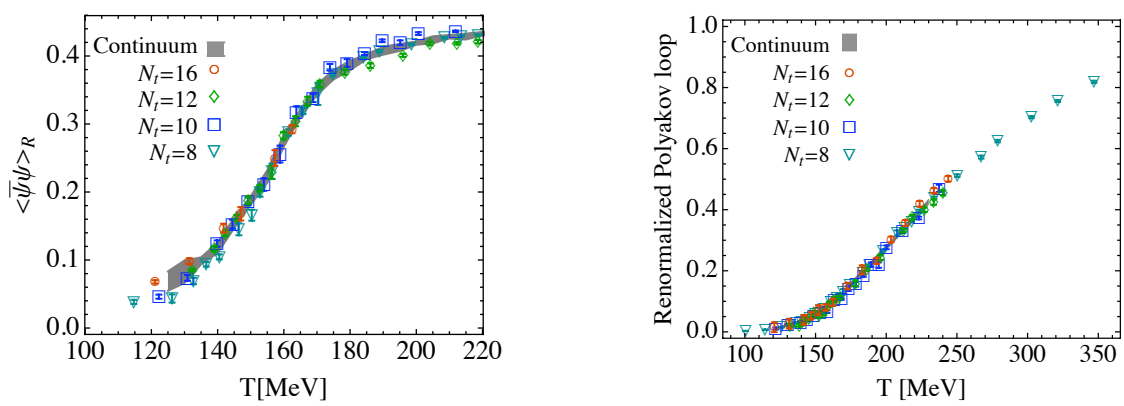

Figure 1: Left: The renormalized chiral condensate. We used the inflection point to define a transition temperature 155(3)(3) MeV. Right: the renormalized Polyakov loop. Although one can precisely measure its temperature dependence, one cannot easily pinpoint an inflection point. The shape of the Polyakov loop curve, as well as any derived transition temperature depends on the details of the renormalization scheme [9]10].

There are other observables that indicate deconfinement. The normalized energy density $\epsilon / T^{4}$ has an inflection point at 157(4)(3) MeV, for the trace anomaly we have 154(4)(3) MeV. The speed of sound has a minimum at around $\sim 145(5) \mathrm{MeV}$. These temperatures cover roughly the same range as we find from chiral observables. From the strange susceptibility, which characterizes the deconfinement of the strange degree of freedom we have a somewhat higher inflection point. Of course, in a broad crossover the definition of the most singular point is also ambiguous.

The concept of an identical chiral and deconfinement transition temperature was promoted by 

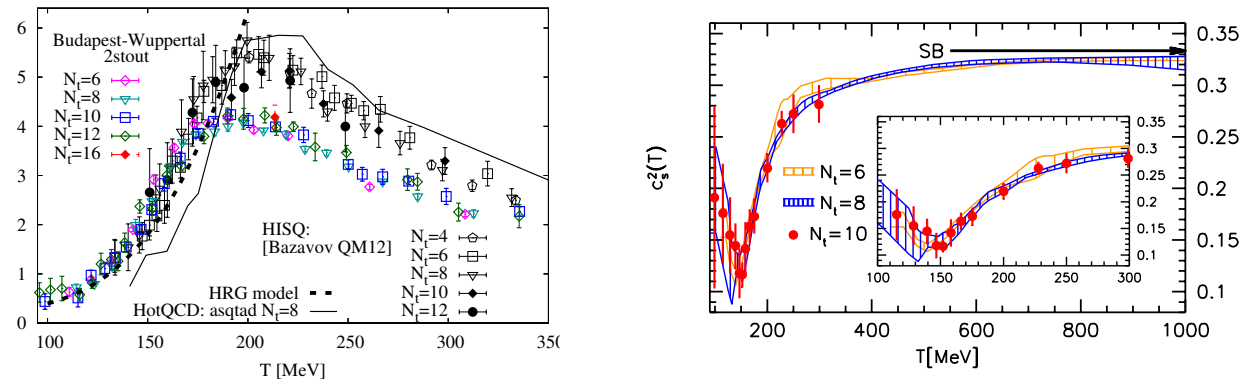

Figure 2: Left: The trace anomaly at several lattice spacings, either with 2stout action of the Wuppertal-Budapest collaboration [15 16], or with the HISQ action of the HotQCD collaboration [17]. For comparison we show the Hadron Gas Resonance model's prediction as well as the historic asqtad data of Ref. [11]. The use of the HISQ action has significantly reduced the discrepancy between the two collaborations. Right: The speed of sound by the WuppertalBudapest collaboration at three lattice spacings [15] (these data are not yet available from HISQ calculations).

early lattice data [12], that positioned the peak of the chiral and the Polyakov loop susceptibilities at the same temperature: $T_{c}$. Lacking resources, only theories with heavier quarks were simulated at the time. Results with somewhat smaller quark masses on coarse lattices confirmed this picture [13]. Solutions to two-flavor QCD based on the functional renormalization group have shown the same conclusion [14]. In full (2+1 flavor) QCD, however, we see a very broad transition. Thus the transition temperatures based on various observables generally do not coincide, they have their values in this broad transition region.

\section{Equation of state}

The pressure and energy density of the quark gluon plasma is one of the key targets of lattice QCD simulations. These form the equation of state, which is required to close the equations of relativistic hydrodynamics [18].

Early works on the QCD equation of state were dominated by quenched results [19, 20]. These were recently extended in range and precision, so that a connection to perturbation theory could be made [21]. The calculations were also extended towards larger gauge groups [22]. For several years we have seen various exploratory studies with non-physical or not renormalized quark masses. Since Ref. [8] physical simulations have been feasible. In staggered simulations discretization effects raise the mass of the hadrons, and through this $T_{c}$ appears to be higher, and the energy and entropy density function is found steeper than physical, and the trace anomaly shows a higher peak. This is an artefact ("taste-breaking") which is absent after continuum extrapolation, its impact on coarse lattices and older actions (like asqtad) was emphasized in Refs. [9, 10, 23]. The Wuppertal-Budapest collaboration exploited the favourable taste-breaking features of the stout action and calculated a continuum estimate of the equation of state [15] based on $N_{t}=6,8$ and 10 runs. HotQCD used the asqtad and p4 actions at $N_{t}=6$ and 8 [11, 24], though these were later found to be incompatible with results with the better HISQ action [17]. For a comprehensive review see Ref. [25].

At this conference, we heard two contributions discussing the equation of state. The WuppertalBudapest collaboration has updated its continuum estimate of 2010 [15] by adding a finer dataset $\left(N_{t}=12\right)$ and an even finer check point $\left(N_{t}=16\right)$ and using these in a continuum extrapolation 

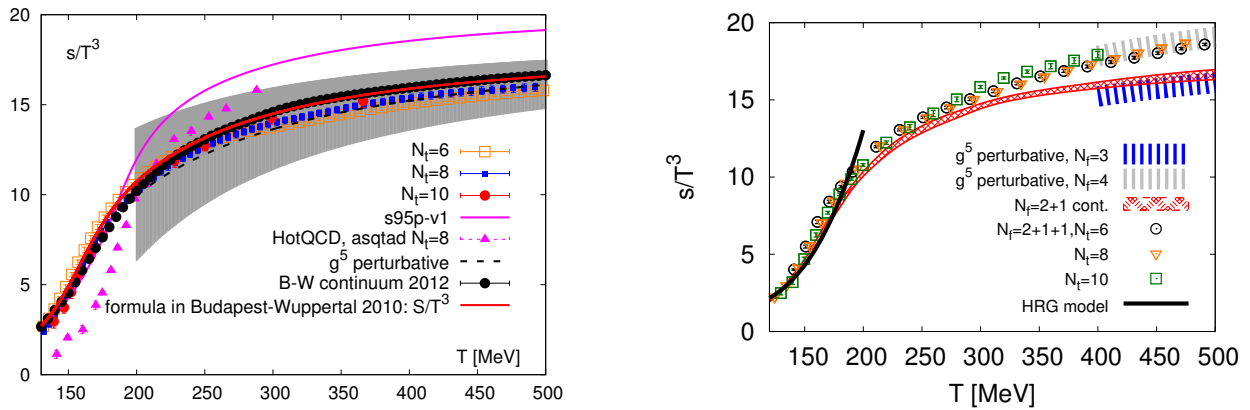

Figure 3: The entropy density as a function of temperature. Left: We show the lattice data by the Wuppertal-Budapest collaboration $\left(N_{t}=6,8\right.$ and 10) together with the parametrization (solid red line) of these data sets published in Ref. [15]. The black dots show the result of a recent continuum extrapolation based on increased statistics and finer lattices $\left(N_{t}=\right.$ 12). We see almost no deviation from the earlier parametrization. Since there is no HISQ data for the entropy density yet we plotted HotQCD's asqtad data set at $N_{t}=8$ for comparison (magenta triangles) [11]. As discussed already (Fig. 2], this set features a higher-than-physical transition temperature and thus it shows steeper equation of state and higher peak in the trace anomaly. As remedy, the s95p parametrization was introduced [23] by replacing lattice data in and below the transition range by the Hadron Resonance Gas prediction. By this, the slope of the entropy curve was left unchanged, but the whole curve was shifted up towards the Stefan-Boltzmann limit. Right: The contribution of the charm sea quark to the entropy density. The Wuppertal-Budapest collaboration's data with $N_{t}=6,8$ and 10 is shown for dynamical $2+1+1$ flavor simulations together with its charmless continuum data and the respective perturbative estimates [16].

[16]. A. Bazavov (HotQCD collaboration) has presented data at $N_{t}=4,6,8,10$ and 12 [17]. First, the trace anomaly was obtained form the lattice simulations. Then, the other quantities, like pressure, entropy density or speed of sound can be derived using thermodynamic relations. The results are summarized in Fig. 2 and 3. The missing contribution of the charm quark has been estimated in C. Ratti's talk, by actually performing new $2+1+1$ flavor simulations (see Fig. 3/right). The charm's contribution to the pressure or energy density was found to be relevant from about 250-300 MeV temperature [16].

\section{Phase diagram}

The transition line in the $T-\mu_{B}$ phase diagram at small chemical potentials is necessarily a line of crossover, with different width and curvature for various observables. Direct Monte-Carlo simulations of the four-dimensional quantum field theory at finite baryo-chemical potential are at present not possible. Nevertheless, starting with Ref. [29] a renewed interest has been seen for $\mu_{B}>0$ questions in lattice QCD, and several indirect strategies exist [30, 31].

The curvature of the transition line in full QCD has been found very small by recent lattice simulations. In Ref. [6] simulations on $N_{t}=8$ lattices were used to map the chiral condensate and its $\mu_{B}^{2}$ dependence onto a universal $\mathrm{O}(4)$ scaling behaviour as a function of the quark mass. Evaluating the fits in the physical point gave $\kappa=0.0066(2)(4)$, with $\kappa=-T_{c} d T_{c}\left(\mu_{B}^{2}\right) /\left.d \mu_{B}^{2}\right|_{\mu_{B}=0}$. The continuum result has been calculated by the Wuppertal-Budapest collaboration [26], with simulations explicitly in the physical point. Their result was $\kappa=0.0066(20)$. In this work, a similar analysis have been made for the strange susceptibility, there the curvature was found to be $0.0089(14)$. In addition, the width of the transition has also been extrapolated to $\mu_{B}>0$. This showed that the transition is not getting stronger with increasing chemical potential, but rather it stays a crossover (see Fig. 4/left). 

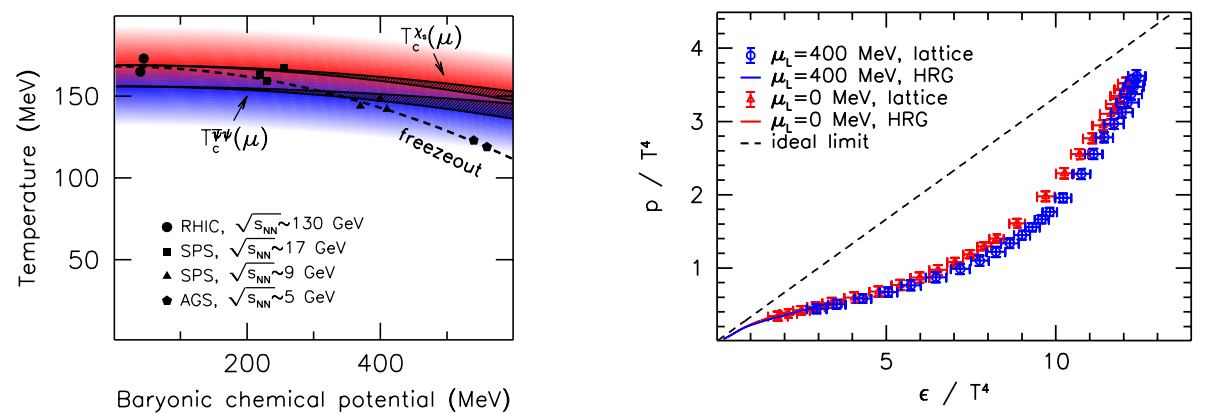

Figure 4: Left: The QCD phase diagram at small chemical potentials [26]. The transition temperature is plotted to leading order in $\mu_{B}^{2} . T_{c}^{\bar{\psi} \psi}$ was extracted from the chiral condensate, and $T_{c}^{\chi_{s}}$ is derived from the strange susceptibility. The solid bands represent the statistical uncertainty, while the color bands stand for the measured width of the transition. At this level of the expansion, there is no indication for the transition getting stronger with increasing $\mu_{B}$. The dashed line show the chemical freeze-out curve of Ref. [27]. Right: Like $T_{c}$, the equation of state can also be extrapolated to finite $\mu_{B}$. Here $\mu_{L}$ refers to the baryo-chemical potential while the mean strageness was kept zero to linear order. For a detailed discussion of these continuum results and a simple parametrization, see Ref. [28].

This latter conclusion is in line with the work by Philipsen and de Forcrand claiming the absence of a critical endpoint, at least in the range where analytical continuation or Taylor expansion can work [32]. This result was often confronted with the critical point found by Fodor and Katz [33]. Note, however, that at small chemical potentials even Ref. [33] finds a slightly weakening transition, this trend then reverses at higher $\mu_{B}$, where the available Taylor expansion from imaginary $\mu_{B}$ no longer works. As the authors also emphasize, these results [32, 33] came from coarse lattices. A continuum limit has not yet been feasible (because of the high computational costs), which leaves us without any conclusive lattice evidence for a critical endpoint or a first order line at high $\mu_{B}$.

Having no singularity in a large range for chemical potentials, the equation of state, too, can be extrapolated to $\mu_{B}>0$. By integrating the trace anomaly we have $\log Z$ at the $\mu_{B}=0$. The baryon number susceptibility $\sim \partial^{2} \log Z /\left.\left(\partial \mu_{B}\right)^{2}\right|_{\mu_{B}=0}$ is also accessible from lattice, thus, a linear expansion is possible. The continuum result was given in Ref. [28] (see Fig. 4).

We finally discuss the effect of a magnetic field. The strong magnetic fields in non-central heavy ion collisions [36] motivated the study of the QCD transition in the presence of an electromagnetic background. A $B$ field is easily implemented on the lattice [37, 38]. Based on models (e.g. PNJL [39]) and exploratory lattice simulations [37] the expectation was to see an increase in $T_{c}$ with growing magnetic field. As a joint effort of the Budapest, Regensburg and Wuppertal lattice groups $T_{c}$ was calculated with physical quark masses in the continuum limit. $T_{c}$ was shown to decrease with $B$ (see Fig. 5/left), while the transition was still a crossover [34]. To show the role played by the quark mass, an auxiliary data set was taken with higher hadron masses. Then the trend was reversed, and the earlier (apparently contradicting) lattice result [37] was reproduced. To explain the behaviour seen in the PNJL model the same group calculated the chiral condensate at $B>0$ and $T>0$, and highlighted the differences between lattice data and the model's assumptions [35] (see Fig. 5) right). 

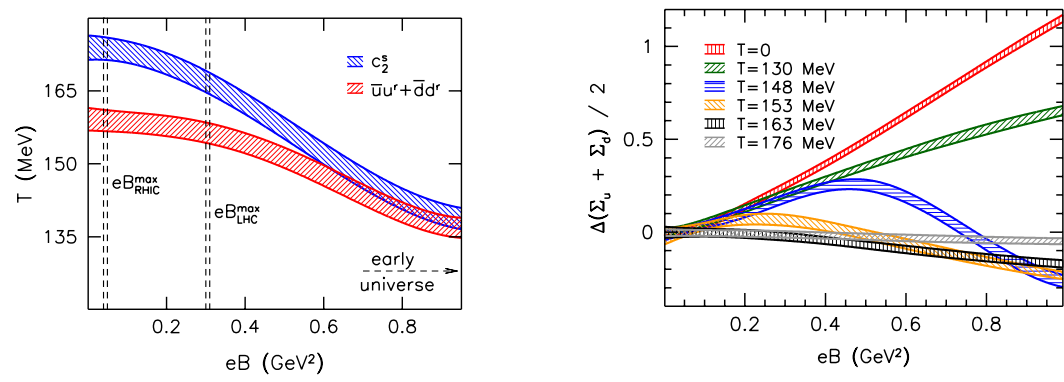

Figure 5: Left: The inflection point of the chiral condensate and of the strange susceptibility. The cross-over temperature decreases with a magnetic field, independently of its definition [34]. Right: The behaviour of the chiral condensate as a function of the magnetic field at various temperatures [35]. The magnetic catalysis at low $T$ turns into a non-monotonic behaviour in transition region.

\section{Fluctuations of conserved charges}

Correlations and fluctuations of conserved charges have been proposed long ago to signal the transition [40, 41]. At high temperatures fluctuations are expected to approach the ideal gas limit. On the other hand, in the low-temperature phase they are expected to be small since quarks are confined and the only states with non-zero quark number have large masses. Agreement with the Hadron Resonance Gas (HRG) model predictions is expected in this phase.

In the past year both the Wuppertal-Budapest [42] and HotQCD [43] collaborations published results for the second order fluctuations, i.e. the second derivatives of the free energy with respect to chemical potentials of various conserved charges, like baryon number, electric charge or strangeness.

The lattice results are always normalized to volume and the respective power of temperature. E.g. the diagonal charge and the off-diagonal charge-baryon correlators are defined by

$$
\chi_{2}^{Q}=\frac{1}{V T^{3}} \frac{\partial^{2} \log Z}{\left(\partial \mu_{Q} / T\right)^{2}}, \quad \chi_{11}^{B Q}=\frac{1}{V T^{3}} \frac{\partial^{2} \log Z}{\left(\partial \mu_{Q} / T\right)\left(\partial \mu_{B} / T\right)} .
$$

The experimentally more interesting observables are ratios of these derivatives, where the unknown volume factor cancels. To describe the freeze-out parameters ( $T$ and $\left.\mu_{B}\right)$ specific ratios have been introduced and calculated (e.g. skewness / mean of the net charge yield) using the coarser data sets of the HISQ action $\left(N_{t}=6,8\right)$ in Ref. [43].

The charge kurtosis $\times$ variance could also be used as a thermometer, and was in the focus of C. Schmidt's talk [45]. In Fig. 7] we plot the temperature dependence of $\chi_{4}^{Q} / \chi_{2}^{Q}$ at zero chemical potential from both the BNL-Bielefeld group and the Wuppertal-Budapest collaboration. While we witness steady progress in the analysis of experimental data towards the precise measurement of second and higher order fluctuations [46, 47, 48], lattice methods are increasingly successful in higher derivatives, which will finally enable the extrapolation of the kurtosis data towards finite chemical potential.

\section{Summary}

We discussed a selection of the recent developments in bulk thermodynamics, focusing on those results with physical quark masses close to or in the continuum limit. For technical reasons, 

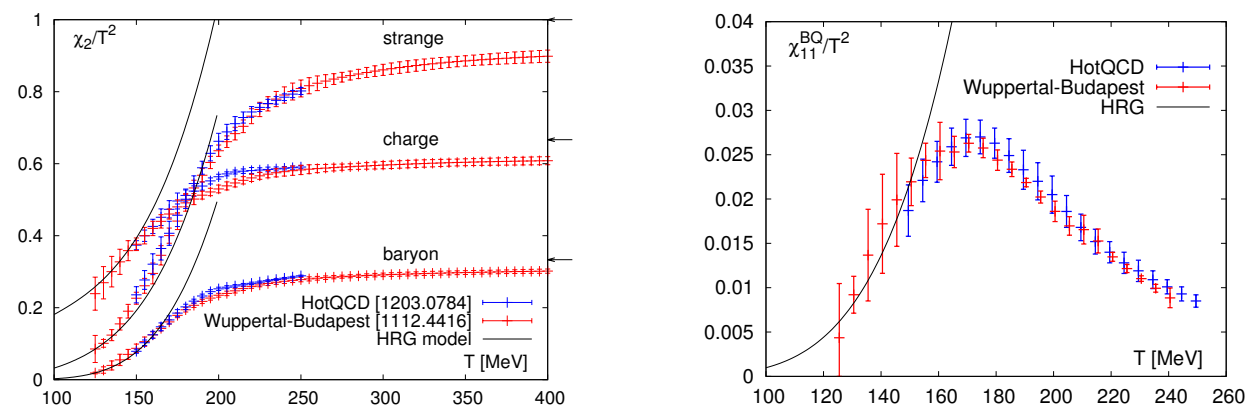

Figure 6: Diagonal and off-diagonal correlators of conserved charges as a function of temperature. Only continuum extrapolated lattice data are shown. There is a good agreement between HotQCD [43] and Wuppertal-Budapest [42 data, obtained using the HISQ and 2stout actions, respectively. The broader temperature region by the WuppertalBudapest collaboration allows a comparison with the HRG model's prediciton, and was used to benchmark resummation schemes at high temperature [44].

all these results were obtained using the staggered formalism. The independence of the results with respect to the applied discretization scheme is an important consistency requirement of lattice QCD, and to this end there have been recent checks with overlap [49], domain-wall [50] and Wilson [51] fermions, with the compromise of using heavy pions in these comparisons.

By today we know several aspects of the QCD transition through Euclidean-time observables at zero and small chemical potentials. Now the challenge for lattice QCD is to develop new techniques for extracting real-time physics (e.g. transport coefficients) and to increase the accessible range in $\mu_{B}$, and eventually, to map the phase diagram.

\section{References}

[1] C. Hoelbling, PoS LATTICE 2010 (2010) 011

[2] C. Morningstar and M. J. Peardon, Phys. Rev. D 69 (2004) 054501

[3] E. Follana et al. [HPQCD and UKQCD Collaborations], Phys. Rev. D 75 (2007) 054502

[4] Y. Aoki, G. Endrodi, Z. Fodor, S. D. Katz and K. K. Szabo, Nature 443 (2006) 675

[5] S. Ejiri, et al.Phys. Rev. D 80 (2009) 094505

[6] O. Kaczmarek, et al,Phys. Rev. D 83 (2011) 014504

[7] A. Bazavov, et al.Phys. Rev. D 85 (2012) 054503

[8] Y. Aoki, Z. Fodor, S. D. Katz and K. K. Szabo, JHEP 0601 (2006) 089

[9] Y. Aoki, Z. Fodor, S. D. Katz and K. K. Szabo, Phys. Lett. B 643 (2006) 46 Y. Aoki, S. Borsanyi, S. Durr, Z. Fodor, S. D. Katz, S. Krieg and K. K. Szabo, JHEP 0906 (2009) 088

[10] S. Borsanyi et al. [Wuppertal-Budapest Collaboration], JHEP 1009 (2010) 073

[11] A. Bazavov et al., Phys. Rev. D 80 (2009) 014504

[12] For an early review of lattice thermodynamics see: F. Karsch, Lect. Notes Phys. 583 (2002) 209 |hep-lat/0106019|

[13] M. Cheng, et al.,Phys. Rev. D 77 (2008) 014511

[14] J. Braun, L. M. Haas, F. Marhauser and J. M. Pawlowski, Phys. Rev. Lett. 106 (2011) 022002

[15] S. Borsanyi et al. JHEP 1011 (2010) 077

[16] C. Ratti (Wuppertal-Budapest collaboration), Proceedings of Quark Matter 2012, Washington D.C.

[17] A. Bazavov (HotQCD collaboration), Proceedings of Quark Matter 2012, Washington D.C. arXiv:1210.6312

[18] P. F. Kolb and U. W. Heinz, In *Hwa, R.C. (ed.) et al.: Quark gluon plasma* 634-714

[19] G. Boyd et al. Nucl. Phys. B 469 (1996) 419

[20] M. Okamoto et al. [CP-PACS Collaboration], Phys. Rev. D 60 (1999) 094510

[21] S. .Borsanyi, G. Endrodi, Z. Fodor, S. D. Katz and K. K. Szabo, JHEP 1207 (2012) 056

[22] See e.g. B. Lucini and M. Panero, arXiv:1210.4997 [hep-th], and references therein 

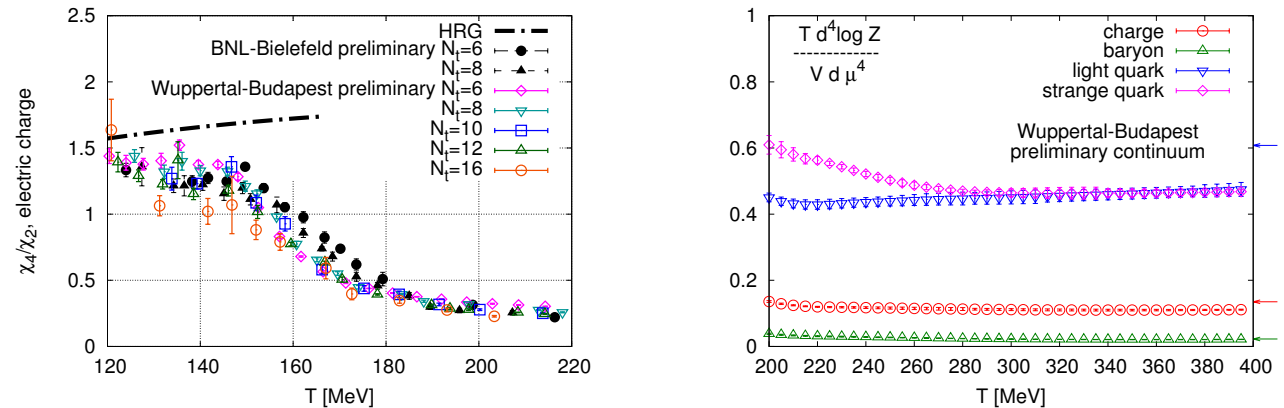

Figure 7: Left: The normalized electric charge kurtosis from lattice QCD: the BNL-Bielefeld collaboration has used the HISQ action on $N_{t}=6$ and 8 lattices [45], the Wuppertal-Budapest collaboration shows results at $N_{t}=6,8,10,12$ and 16. From the plot we see that the use of fine lattice is essential for a controlled continuum limit. At present data indicates deviations from the Hadron Resonance Gas model's prediction even below $T_{c}$. Right: At high temperatures the Wuppertal-Budapest collaboration has attempted a preliminary continuum extrapolation for $\chi_{4}$ for electric charge, strangeness and baryon number. The difference we see between light and strange quark's behaviour indicates that the mass of the strange quark is still noticeable up to about $260 \mathrm{MeV}$ temperature.

[23] P. Huovinen and P. Petreczky, Nucl. Phys. A 837 (2010) 26

[24] M. Cheng et al. Phys. Rev. D 81 (2010) 054504

[25] O. Philipsen, arXiv:1207.5999 [hep-lat].

[26] G. Endrodi, Z. Fodor, S. D. Katz and K. K. Szabo, JHEP 1104 (2011) 001

[27] J. Cleymans and K. Redlich, Phys. Rev. Lett. 81 (1998) 5284

[28] S. .Borsanyi, G. Endrodi, Z. Fodor, S. D. Katz, S. Krieg, C. Ratti and K. K. Szabo, JHEP 1208 (2012) 053

[29] Z. Fodor and S. D. Katz, Phys. Lett. B 534 (2002) 87

[30] Z. Fodor and S. D. Katz, arXiv:0908.3341 [hep-ph].

[31] For a review, see: O. Philipsen, arXiv:1009.4089 [hep-lat].

[32] P. de Forcrand and O. Philipsen, JHEP 0701 (2007) 077

[33] Z. Fodor and S. D. Katz, JHEP 0404 (2004) 050

[34] G. S. Bali et al., JHEP 1202 (2012) 044

[35] G. S. Bali, F. Bruckmann, G. Endrodi, Z. Fodor, S. D. Katz and A. Schafer,arXiv:1206.4205 [hep-lat].

[36] V. Skokov, A. Y. .Illarionov and V. Toneev, Int. J. Mod. Phys. A 24 (2009) 5925

[37] P. Cea, L. Cosmai and M. D’Elia, JHEP 0712 (2007) 097

[38] E. -M. Ilgenfritz et al. Phys. Rev. D 85 (2012) 114504

[39] R. Gatto and M. Ruggieri, Phys. Rev. D 83 (2011) 034016

[40] S. Jeon and V. Koch, Phys. Rev. Lett. 85, 2076 (2000)

[41] M. Asakawa, U. W. Heinz and B. Muller, Phys. Rev. Lett. 85, 2072 (2000)

[42] S. Borsanyi, Z. Fodor, S. D. Katz, S. Krieg, C. Ratti and K. Szabo, JHEP 1201 (2012) 138

[43] A. Bazavov et al. [HotQCD Collaboration], Phys. Rev. D 86 (2012) 034509

[44] J. O. Andersen, S. Mogliacci, N. Su and A. Vuorinen, arXiv:1210.0912 [hep-ph].

[45] C. Schmidt (BNL-Bielefeld collaboration), Proceedings of Quark Matter 2012, Washington D.C.

[46] J. T. Mitchell (PHENIX collaboration), Proceedings of Quark Matter 2012, Washington D.C.

[47] D. McDonald (STAR collaboration), Proceedings of Quark Matter 2012, Washington D.C.

[48] X. Luo (STAR collaboration), Proceedings of Quark Matter 2012, Washington D.C. arXiv:1210.5573] [nucl-ex].

[49] S. Borsanyi, Y. Delgado, S. Durr, Z. Fodor, S. D. Katz, S. Krieg, T. Lippert and D. Nogradi et al., Phys. Lett. B 713 (2012) 342

[50] A. Bazavov, et al. [HotQCD Collaboration], arXiv:1205.3535 [hep-lat].

[51] S. Borsanyi, S. Durr, Z. Fodor, C. Hoelbling, S. D. Katz, S. Krieg, D. Nogradi and K. K. Szabo et al., JHEP 1208 (2012) 126 